\title{
Thymic microenvironments, 3-D versus 2-D?
}

\author{
Willem van Ewijk*, BaoPing Wangt, Georg H ollähderł, H iroshi Kawamoto§, \\ Eugenia Spanopoulou", Manami Itoi", Takashi Amagai", Yu-Fei Jiang*† \\ Wilfred T.V. Germeraad**, Wei-Fung Chen $†$ t and Yoshimoto Katsura§
}

Lympho-stromal interactions in the thymus crucially de termine the fate of developing $T$ cells. Epithelial cells, interdigitating reticular cells, macrophages and fibroblasts all play a role in the shaping of the $T$ cell repertoire. Recently published evidence shows that lympho-stromal interaction acts bi-directional. Developing $T$ cell themselves, at different stages of differentiation, control the microarchitecture of thymic microenvironments, a phenomenon designated as 'crosstalk'. This paper reviews experiments showing that developing $T$ cells crosstalk to different thymic epithelial cells in a stepwise fashion. In this way, correctly organized thymic microenvironments guarantee normal thymopoiesis.

Key words: thymus / microenvironments / epithelial cells / crosstalk / lympho-stromal interaction

(C)1999 Academic Press

\section{Introduction}

The thymus is an unique lymphoid organ, because, in contrast to secondary lymphoid organs, its stroma is, by and large, created by epithelial cells instead of mesenchyme derived cells. In origin, around Ed10 in development, the thymus in the mouse derives both

From the *Department of Immunology, Erasmus University Rotterdam, Postbox 1738, 3000 DR R otterdam, The N etherlands, the $\dagger$ Beth I srae H ospital, $\mathrm{H}$ arvard University, Boston, USA, the ‡Department of Research, Pediatric Immunology, Kantonsspital, Basel, Switzerland, the $\S$ D epartment of I mmun ology, Chest Disease Research Institute, K yoto University, K yoto, Japan, the "Ruttenberg Cancer Center, $\mathrm{H}$ oward $\mathrm{H}$ ughes $\mathrm{M}$ edical Institute, Mount Sinai School of Medicine, New York, USA, the "Department of Immunology and $\mathrm{M}$ icrobiology, $\mathrm{Meji}$ University of Oriental M edicine, Kyoto, Japan, the ** Department of Immunology, University $\mathrm{H}$ ospital, Utrecht, The $\mathrm{N}$ etherlands and $t \dagger \mathrm{Department}$ of Immunology, Beijing M edical University, Bejjing, China

(C)1999 A cademic Press

$1044-5323 / 99 / 010057+08 \$ 30.00 / 0$ from contributions of the endoderm of the third pharyngeal pouch, the ectoderm of the third pharyngeal cleft and mesenchymal cells, originating in the neural crest region. ${ }^{1-3}$ This thymic 'anlage' is populated by IKAROS ${ }^{+}$lymphoid progenitor cells from Ed11 onwards (Itoi, M. and Amagai, T., manuscript in preparation). Recently, it has been shown that intercellular communication between various cell types controls the development of the typical architecture of the thymic stroma. Already early in ontogeny, interaction between mesenchymal cells and epithelial cells appears essential for thymic organogenesis., ${ }^{3,4}$ Later on, developing lymphoid cells at various stages of differentiation influence the typical organization of thymic microenvironments. ${ }^{5,6}$

The nature of these intercellular communication signals is at present unclear, but one could speculate that such signals lead to activation of epithelial specific transcription factors regulating the thymus-specific differentiation of epithelial cells. ${ }^{7}$

\section{What makes the thymic stroma unique?}

Histological, electron-microscopical, and immunohis tological studies have revealed the typical organization of the thymic stroma. ${ }^{8-12}$ Basically, the thymic stroma consists of epithelial cells, which both in the cortex and in the medulla form a specific three-dimensionally (3-D) oriented network (Figure 1). From a histological point of view, this particular type of organization of the thymic stroma is peculiar. In the majority of visceral organs, epithelial cells are placed on a basement membrane, forming sheets of cells which create borders in and between organs. Such 'classical' epithelial cells organize themselves basically in a two-dimensionally (2-D) oriented fashion. Thus, the unique character of the thymic epithelium lies in the extra, third, dimension, which permits thymic epithelial cells to create a spongelike stroma. This 


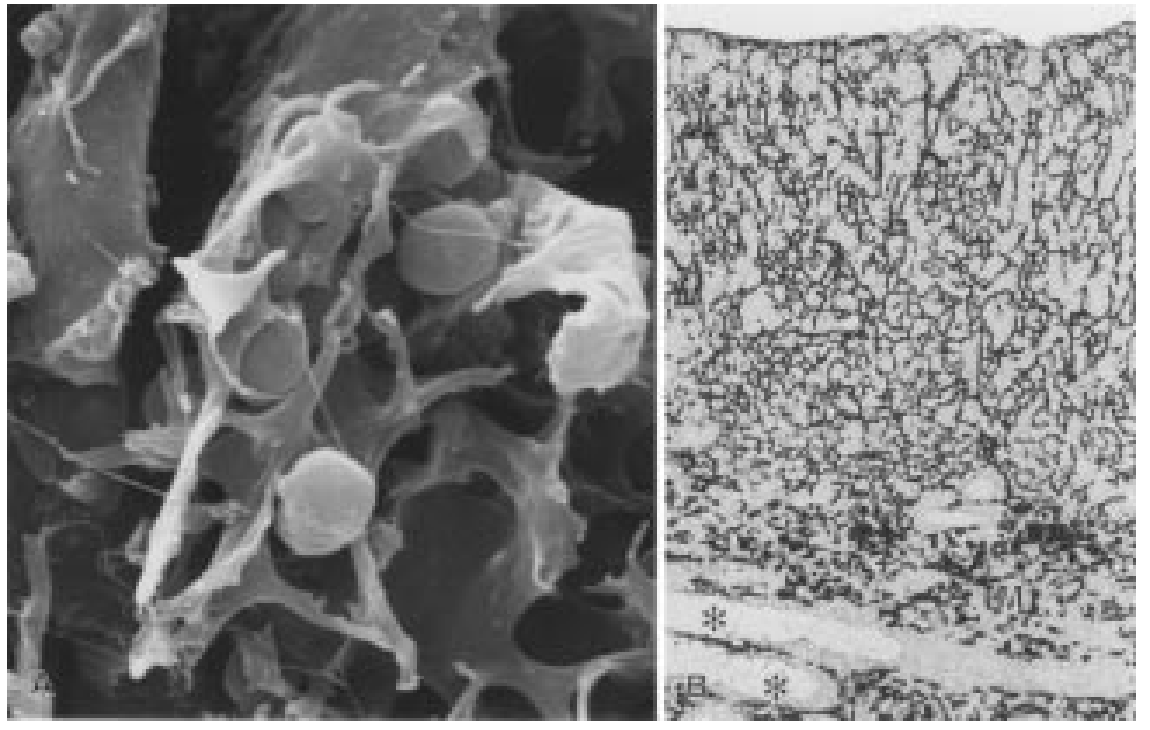

Figure 1. (a) Scanning electronmicroscopic micrograph of thymocytes and cortical epithelial cells. Cortical epithelial cells form a 3-D network structure allowing migration of developing $T$ cells through the thymic stroma $(\times 3300)$. (b) The thymic epithelial stroma visualized with the single chain antibody TB4-20. Epithelial cells in the outer cortical region are oriented perpendicular to the thymic capsule, promoting a lymphocyte flow from cortex towards medulla ( $c=$ cortex, $\mathrm{m}=$ medulla). Venules are indicated by asterisks $(\times 85)$.
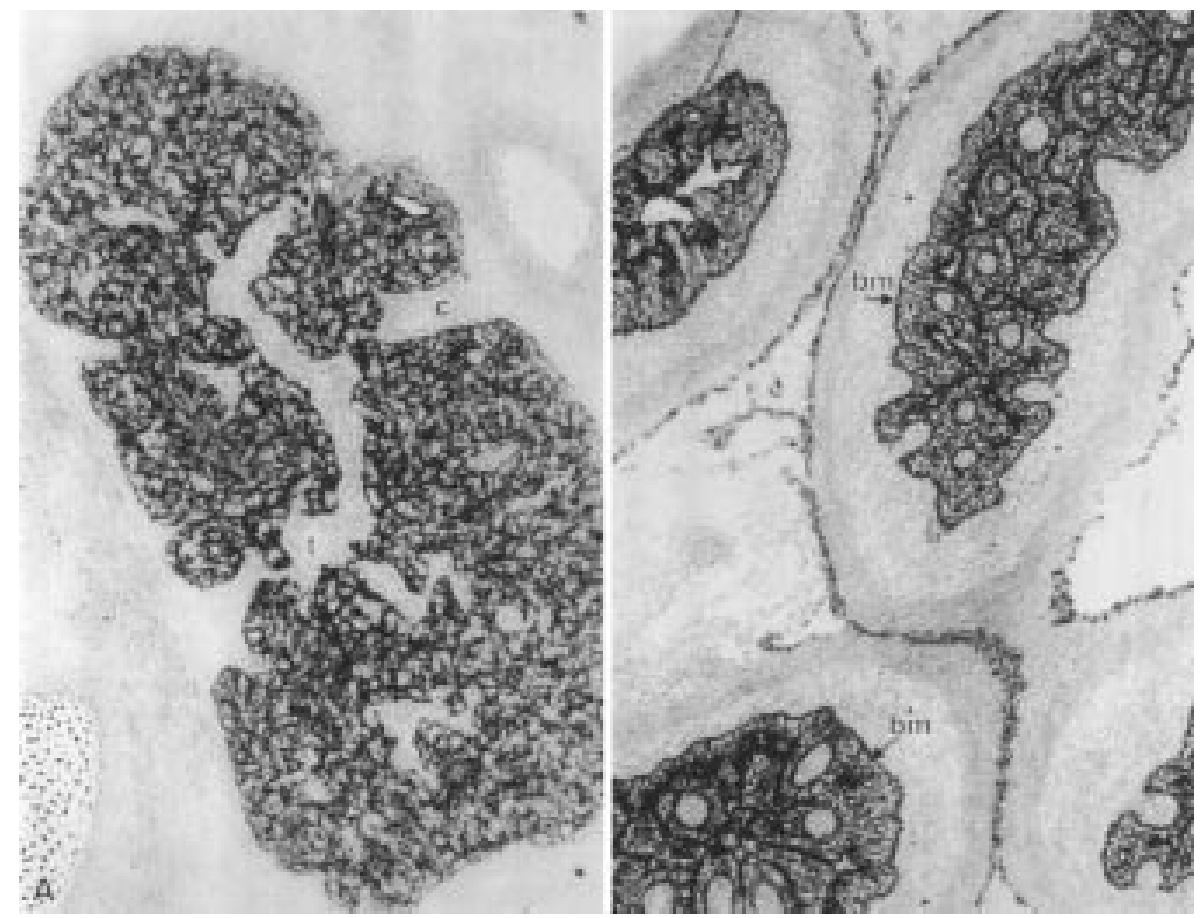

Figure 2. (a) The thymic stroma, identified by the single chain antibody TB4-20, in the thymus at day 14 of gestation al ready shows a sponge-like 3-D organized structure ( $c=$ capsule, $t=$ trabecula; $\times 120)$. (b) Intestinal epithelial cells, stained by TB4-20, $(\times 90)$ at day 14 of gestation are organized in 2-D oriented sheets, positioned on a basement membrane (bm; $\times 90)$. 
typical organization is already created early in ontogeny, from day 14 in gestation onwards (see also Figure 2).

Recent experiments have shown that this typical 3-D organization, is, at least in part, controlled for by developing $\mathrm{T}$ cells, a phenomenon designated as 'thymic crosstalk'.5 Apparently, thymic crosstalk occurs both in the cortex as well as in the medulla, but different lymphoid subpopulations appear to regulate the organization of the thymic stroma in these compartments. We will first briefly mention experiments on crosstalk in the medulla and then review more recent experiments on crosstalk in the cortex.

\section{Crosstalk in the thymic medulla}

Earlier published experiments, performed in close collaboration with Al Singer and Wendy Shores at $\mathrm{NIH}^{13}$ for the first time demonstrated the influence of developing thymocytes on the architecture of thymic microenvironments in SCID mice and in bone marrow transplanted SCID mice. The thymus in SCID mice is abnormal in the sense that it shows a well developed thymic cortex, but medullas are virtually absent. In asking whether this aberrant thymus still could support $\mathrm{T}$ cell development, we transplanted SCID mice with bone marrow cells derived from normal mice. Indeed, such transplanted SCID mice showed normal $T$ cell development to the level of single $\mathrm{CD}^{+}{ }^{+}$and $\mathrm{CD}^{+}{ }^{+} \mathrm{T}$ cells, expressing $\mathrm{T}$ cell receptors (TCR) at high density. Surprisingly, immunohistology of the thymus learned that, after bone marrow transplantation, the thymic stroma completely reorganized in the same manner as found in the thymus of normal mice. Thus, well localized medullas reappeared, revealing for the first time the plasticity of the thymic stroma. Rather than being a static structure, the thymic stroma can be remodeled, and 'mature' developing thymocytes which reside in between thymic medullary epithelial cells, influence the typical organization of medullary epithelial cells, resulting in the formation of correctly organized medullas. ${ }^{13,14}$ This notion is based on immunohistological studies, using monoclonal antibodies specific for thymic cortical and medullary epithelial cells, respectively (ER-TR4 and ER-TR $5^{10}$ ).

The actual mechanism used by developing $T$ cells to reorganize the thymic medulla is at present still not well understood, but transplantation of SCID mice with mature peripheral $\mathrm{T}$ cells, ${ }^{15}$ and crossings of SCID mice to $T$ cell receptor transgenic mice, ${ }^{16}$ showed that mature T cells, and also late DP thymo- cytes were responsible for the reorganization of the thymic medulla. Similar data were obtained in RAG null mice and RAG null mice crossed to TCR transgenic mice (Spanopoulou, E. and van Ewijk, W., unpublished result; Figure 3). More mature thymocytes reside within the thymus in close proximity to medullary epithelial cells, and it seems likely that cell-cell interaction or short-range operating factors secreted by these relatively mature $T$ cells regulate the expansion and differentiation of thymic medullary epithelial cells.

The finding that reconstitution of RAG null mice with normal bone marrow leads to proliferation of cortical epithelial cells, but not medullary epithelial cells, ${ }^{17}$ raises the interesting possibility that medullary epithelial cells derive from precursor epithelial cells in the cortex. On the other hand, ER-TR4 ${ }^{+}$, ER-TR5 ${ }^{+}$ 'double positive' epithelial cells have been described in the thymus, ${ }^{18}$ indicating the presence of epithelial 'stem' cells in the thymus with differentiation potential both towards cortical and medullary epithelial cells. So, the exact relationship between epithelial cells in the cortex and medulla remains at present unresolved, but clearly, subsets of developing, mature thymocytes regulate the expansion and positioning of epithelial cells in the medulla.

\section{Crosstalk in the thymic cortex}

If thymic crosstalk exists in the thymic medulla, does it also occur in the thymic cortex? The answer to this question is positive. Two different experimental models have clearly shown that developing T cells control the architecture of cortical thymic microenvironments. The first model is based on in vivo studies applying genetically manipulated mice which show a very early block in T cell development. This model was developed in the group of Dr Cox Terhorst at Harvard University. ${ }^{19}$ The second experimental model is based on in vitro cultured fetal thymic explants, and was developed in the group of $\mathrm{Dr}$ Yoshimoto Katsura at Kyoto University. ${ }^{20}$

Recently, we showed that mice which overexpress the human $\operatorname{CD} 3 \varepsilon$ gene as a transgene show an arrest in $T$ cell development at an early level, characterized by absence of CD3, CD4, CD8 ('triple negative'; TN) and presence of CD44, but not CD25. Such CD3 $\varepsilon$ transgenic mice showed aberrant thymic microenvironments, characterized by a complete absence of thymic medullas; a few remaining ER-TR5 ${ }^{+}$ medullary epithelial cells were found scattered throughout the thymus. ${ }^{6}$ Besides, cortical epithelial 


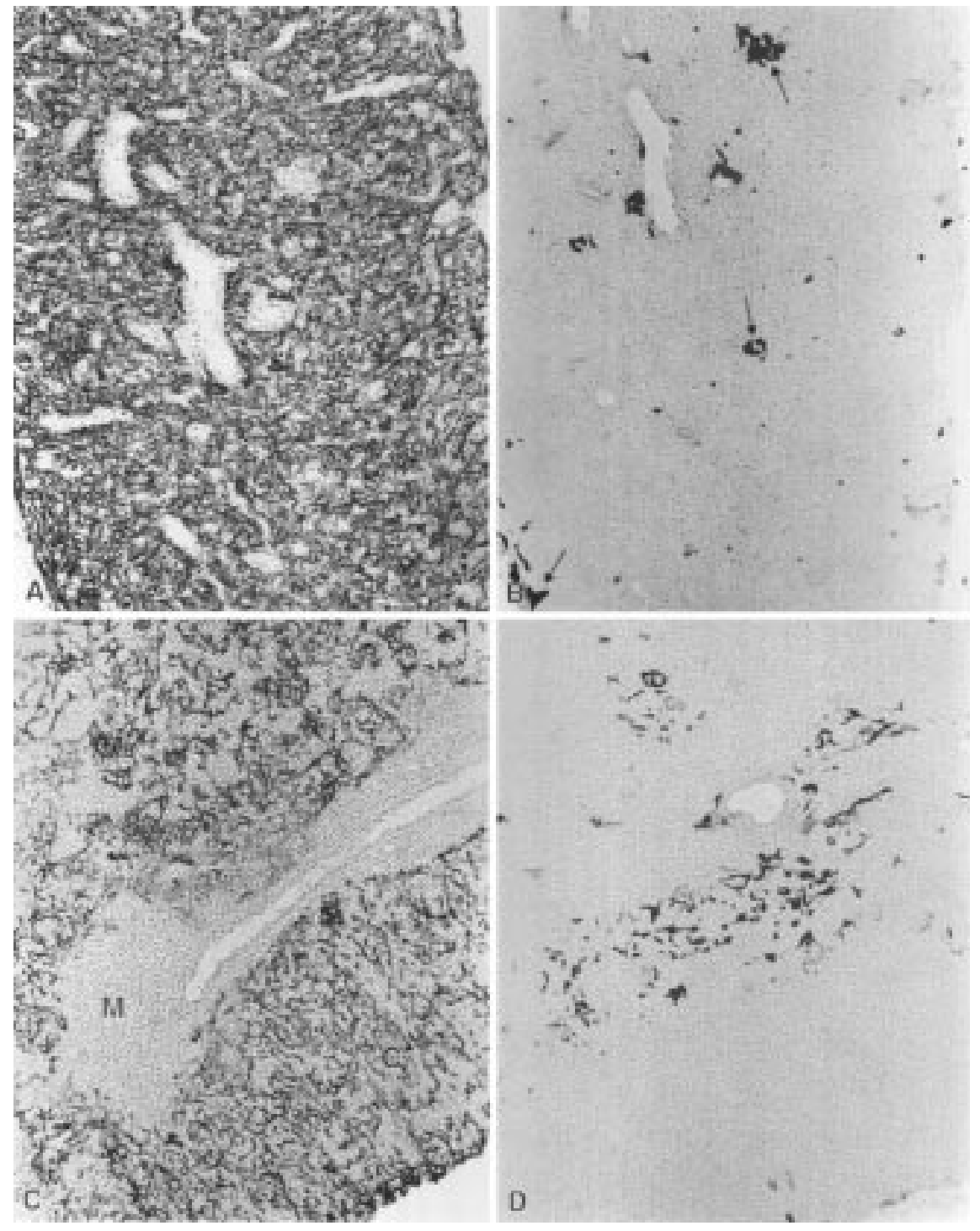

Figure 3. The thymic stroma in RAG null mice $(a, d)$ and in RAG null mice crossed to ${ }_{\alpha \beta}$ TCR transgenic mice. ER-TR4 reveals cortical epithelial cells (a,c); ER-TR5 reveals medullary epithelial cells $(b, d)$. Note small patches of medullary cells in the thymus of RAG null mice, whereas in the RAG null $\times$ TCR transgenic mice both cortical and medullary microenvironments are normal (all magnifications are $\times 90$ ).

cells had reorganized themselves. In the normal thymus, cortical epithelial cells are oriented perpendicularly towards the thymic capsule (see also Figure 1), thereby creating migration pathways for developing thymocytes from the cortex towards the medulla. ${ }^{8,9}$ In contrast, cortical epithelial cells in the thymus of $\mathrm{CD} 3 \varepsilon$ transgenic mice were found to be oriented parallel to the thymic capsule. Most strikingly, however, was the presence of large cysts in the thymus of these mice (Figure 4). Close morphological inspec- tion of such cysts revealed that 'classical' epithelial cells, such as absorptive cells, ciliated cells and goblet cells, all placed on a basement membrane, were present in these cysts (see also Figure 2a). Apparently, in the thymus of $C D 3 \varepsilon$ transgenic mice, the 3-D stromal architecture had turned back into a 2-D organization

The question then arose whether in the $C D 3 \varepsilon$ transgenic mice 2-D could be rebuilt into 3-D by bone marrow transplantation. Initial experiments in 


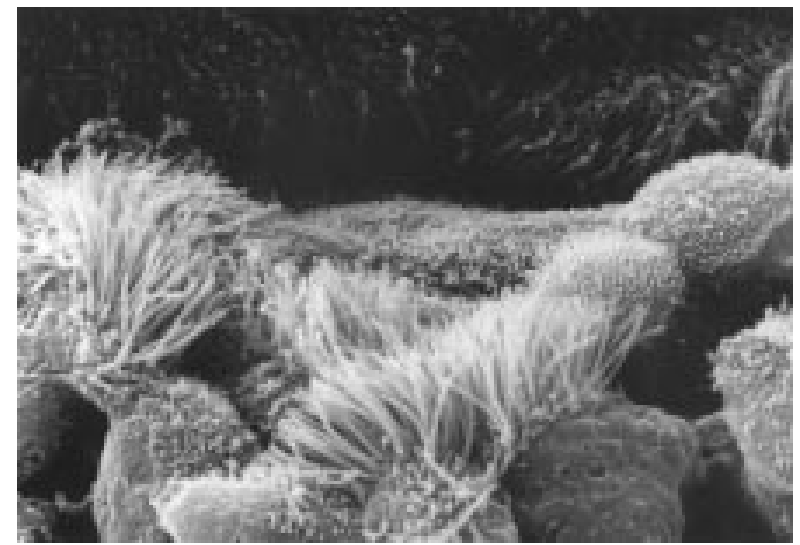

Figure 4. Ciliated epithelial cells lining a cyst in the thymus of $\mathrm{CD} 3 \varepsilon$ transgenic mice. (Scanning electronmicroscopy $\times 1500$.)

which adult CD $3 \varepsilon$ transgenic mice were transplanted with normal bone marrow cells showed only marginal reconstitution of $T$ cell development and $T$ cell function. The thymus in these transplanted mice remained small in size. Furthermore, such mice developed autoimmune disease, indicating a defective selection process in the thymus of these reconstituted mice. $^{6}$

By contrast, transplantation of $\mathrm{CD} 3 \varepsilon$ transgenic mice at a fetal stage in development, using fetal liver cells, generated mice with normal levels of peripheral $T$ cells. Immunohistologically, such mice now also showed completely normal thymic microenvironments ${ }^{6}$.

Several important conclusions were drawn from these experiments. First, similarly to medullary epithelial cells, cortical epithelial cells show plasticity. Second, an arrest at an early stage of T cell development, characterized by absence of CD3, CD4 and CD8, presence of CD44 but absence of CD25 completely blocks development of the tertiary structure of the epithelial network in the thymic cortex. Apparently, absence of signals derived from thymocytes beyond this developmental control point, where TCR $\beta, \gamma$ and $\delta$ gene rearrangements occur, ${ }^{21}$ leads to a 'classical' type of organization, forcing epithelial cells to organize their positioning along a basement membrane, thereby creating thymic cysts. Third, the organization of the cortical epithelial stroma is dependant on control points situated in the early, fetal development period of the thymus. While medullary epithelial cells can reorganize themselves both in the fetal and the adult thymus, cortical epithelial cells can do so only at the fetal stage of development. Apparently, the cortex and the medulla each have different developmental windows allowing reorganization, expansion and positioning of the epithelial cells.

\section{Stepwise control of stromal development}

Recent studies (van Ewijk et $\mathrm{al}^{22}$ ) have further highlighted a stepwise control mechanism of differentiating thymocytes in the development of the thymic stroma. Since SCID mice, but also RAG null mice, show an arrest of developing $T$ cells at the level of TN CD44 ${ }^{-} / \mathrm{CD}_{25} 5^{+}$, a slightly later stage in development compared to that in $\mathrm{CD} 3 \varepsilon$ transgenic mice, and because of the fact that both SCID and RAG null mice show a normal thymic cortex, we asked whether transplantation of $\mathrm{CD} 3 \varepsilon$ transgenic mice with bone marrow cells derived from RAG null mice could restore the thymic stroma up to the level of organization found in the thymus of SCID and RAG null mice. So, the hypothesis was that this type of transplantation should repair cortical microenvironments but not medullary microenvironments. Indeed, and to our satisfaction, we found that RAG null bone marrow transplantation into $\mathrm{CD} 3 \varepsilon$ transgenic mice induced loss of thymic cysts and reappearance of 3-D oriented cortical microenvironments. Moreover, this type of transplantation did not induce thymic ER-TR5 ${ }^{+}$ medullas. An important conclusion from this experiment is that the transition of $\mathrm{TN} \mathrm{CD} 44^{+} / \mathrm{CD} 25^{-}$ thymocytes into $\mathrm{TN} \mathrm{CD} 44^{-} / \mathrm{CD} 25^{+}$thymocytes induces the extra dimension in the orientation of the thymic cortical epithelium.

In terms of cellular organization, this particular experiment also showed two other important points. First, the vascularisation of the thymus of the transplanted mice dramatically increased, and especially in the thymic cortex many new capillaries appeared. Second, a high frequency of thymic nurse cells (TNC ${ }^{23,24}$ ) was found upon bone marrow transplanta tion. Such cells were mainly found in the subcapsular cortex of the thymus in close proximity to the newly developed capillaries. These phenomena further stress, in terms of thymic crosstalk, the importance of the Iymphoid control point where thymocytes shift their phenotype from TN CD $44^{+} / \mathrm{CD} 25^{-}$into TN CD $44^{-} /$CD $25^{+}$.

While transplantation of $\mathrm{CD} 3 \varepsilon$ transgenic mice with normal bone marrow at adult age failed to induce normal thymopoiesis, ${ }^{6}$ transplantation of $\mathrm{RAG}^{\text {null }} \rightarrow \mathrm{CD} 3 \varepsilon$ chimeric mice at adult age with 
normal bone marrow did result in normal $\mathrm{T}$ cell development. Immunohistological analysis of the thymic stroma in the latter type of transplanted mice revealed that now, both cortical and medullary epithelial cells were organized as in the thymus of normal mice. Clearly, these experiments show that the first transplantation of CD3 $\varepsilon$ mice with RAG null bone marrow pre-conditions the thymic stroma for normal $T$ cell development. Moreover, since the first transplantation induced the thymic cortex, while the second transplantation induced the medulla, it follows that developing $T$ cells crosstalk to the thymic epithelial stroma in a stepwise fashion.

Importantly, these results also indicate that the generation of proper cortical microenvironments is a prerequisite for the generation of correct medullary microenvironments. This rule also applies to thymic development in normal mice, since during embryogenesis the thymic cortex appears before the thymic medulla. ${ }^{25}$

\section{Oxygen and $\mathrm{T}$ cell progenitors both control the 3-D organization of the thymic stroma}

The increased angiogenesis observed in the thymus of CD3 $\varepsilon$ transgenic mice after RAG null bone marrow transplantation indicated that the oxygen concentration in the thymus regulates the development of the typical 3-D structure of the thymic stromal network. To analyze the influence of oxygen on the development of the thymic stroma, we recently cultured isolated thymic lobes under conditions of high oxygen supply (HOS) and low oxygen supply $\left(\operatorname{LOS}^{26}\right)$. The culture experiments were performed in the group of Dr Katsura in Kyoto, while the immunohistological analysis was performed in Rotterdam. This study showed that, under HOS conditions, T cells developed to full maturation within a nicely 3-D oriented thymic stroma (Figure 5a). H owever, under LOS conditions, $T$ cell development remained arrested at the TN CD $44^{+} / \mathrm{CD} 25^{-}$stage. Under the latter experimental conditions, the thymic epithelial cells failed to develop their typical 3-D architecture and organized themselves into 2-D oriented thymic cysts (Figure $5 b$ ).

At this point is was not clear whether the loss of the 3-D architecture of the thymic stroma was caused by the inability of the thymocyte progenitors to develop under LOS conditions, by the low oxygen concentration only, or by both factors. To study a direct effect of the oxygen concentration on the organization of the thymic stroma we 'purified' thymic epithelium by deoxyguanosine (d-Guo) treatment. This procedure is known to remove developing $T$ cells from the fetal
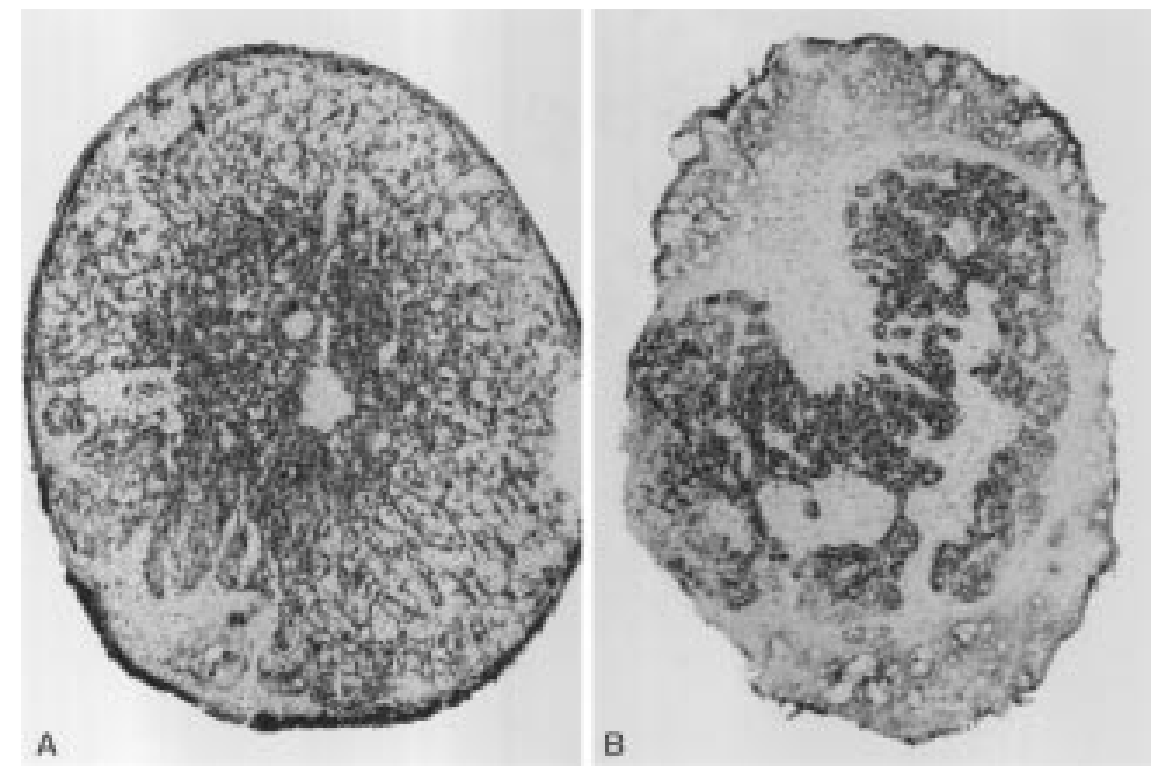

Figure 5. (a) Epithelial cells in Ed14 thymic explants cultured under high oxygen pressure. Note a 3-D organized thymic epithelial network at the periphery of the thymic lobe cultured under high oxygen pressure $(\times 90)$. (b) Under low oxygen pressure, thymic epithelial cells retract their cellular processes. They form 2-D organized sheets positioned on a basement membrane $(\times 90)$. 
thymus. $^{27}$ Immunohistology of d-Guo treated thymi revealed a complete loss of the 3-D architecture. ${ }^{26}$ Culture of such treated thymic lobes under HOS conditions did not result in reappearance of the 3-D architecture. As expected, culture of d-Guo treated lobes under LOS conditions also did not result in a network structure of the thymic stroma. However, in experiments where d-Guo treated lobes were co-cultured with Sca- $1^{10}$ Iymphoid progenitor cells, the 3-D orientation of the thymic stroma was re-established, albeit only under HOS conditions. These results indicate that for proper induction of the 3-D orientation of cortical microenvironments, both a high concentration of oxygen and the actual presence of lymphoid progenitors within the thymus is required. Similar to the above mentioned in vivo experiments with transplanted $\mathrm{CD} 3 \varepsilon$ transgenic mice, these in vitro experiments highlight the importance of the control point in lymphoid development where thymocytes shift their phenotype from TN $\mathrm{CD} 44^{+} / \mathrm{CD} 25^{-}$into TN CD $44^{-} / \mathrm{CD} 25^{+}$. Apparently, this control point is of crucial importance for the development of the tertiary structure of cortical thymic microenvironments.

\section{Directions for future research}

The question is now to be answered which lymphoid derived factors influence the 3-D organization of the thymic stroma. Of interest will be a comparative analysis of the cell surface phenotype of thymocytes shifting from TN CD $44^{+} / \mathrm{CD} 25^{-}$to TN CD $44^{-} / \mathrm{CD} 25^{+}$. Comparative analysis of cytokines (see also ref 28) produced by these two types of thymocytes will provide clues to these mechanism of thymic crosstalk in the cortex. Similarly, both types of analysis should reveal signals important in crosstalk between mature thymocytes and medullary epithelial cells. Most importantly, representational difference analysis ${ }^{7}$ comparing the $\mathrm{CD} 3 \varepsilon$ and RAG thymus should further unravel the nature of molecules which signal differentiation of epithelial cells into the 3-D oriented fashion required for normal thymopoiesis.

\section{Students and teachers}

Finally, the question why the 3-D architecture of the thymic stroma is essential in $\mathrm{T}$ cell development has not yet been answered. The answer may in fact be quite simple. Only in 3-D oriented microenviron- ments, developing $T$ cells, within a time period of approximately 2.5 weeks, ${ }^{29}$ can migrate and contact different types of stromal cells. Moreover, this special organization of thymic epithelial cells allows for the creation of microenvironments with biochemically defined micromilieus, promoting proliferation, differentiation and selection of developing $T$ cells. So, in a way, the thymus is comparable to a (medical?) school. On the one hand, within defined classrooms, the faculty of stromal cells teaches and selects brilliant students. But, on the other hand, what would that faculty be without such brilliant students?

\section{References}

1. O wen JJT, Jenkinson EJ (1984) Early events in T lymphocyte genesis in the fetal thymus. Am J Anat 170:301-310

2. Bockman DE, Kirby ML (1984) Dependence of thymus development on derivatives of the neural crest. Science 223:498-500

3. Itoi M, Amagai T (1998) Inductive role of fibroblastic cell lines in development of the mouse thymus anlage in organ culture. Cell Immunol 183:32-41

4. Auerbach R (1960) Morphogenetic interactions in the development of the mouse thymus gland. Dev Biol 2:271-284

5. van Ewijk W, Shores EW, Singer A (1994) Crosstalk in the mouse thymus. Immunol Today 15:214-217

6. Holländer $G A$, Wang $B$, Nichogiannopoulou $A$, Platenburg PP, van Ewijk W, Burakoff SJ, Gutierrez-Ramos J-C, Terhorst C (1995) Developmental control point in induction of thymic cortex regulated by a subpopulation of prothymocytes. $\mathrm{Na}$ ture 373:350-353

7. Lerner A, Clayton LK, Miroqucki E, Chendler $Y$, Koyasu $S$, Bhan AK, van Ewijk W, Reinherz EL (1996). Cross linking of $T$ cell receptors on double positive thymocytes induces a cytokine-mediated activation process linked to cell death. EMBO । 15:5876-5887

8. van Ewijk W (1988) Cell surface topography of thymic microenvironments. Lab Invest 59:579-590.

9. van Ewijk W (1991) T-Cell differentiation is influenced by thymic microenvironments. Annu Rev Immunol 9:591-615

10. van Vliet E, Melis M, van Ewijk W (1984) Monoclonal antibodies to stromal cell types of the mouse thymus. Eur J Immunol 14:524-529

11. van Vliet $E$, Jenkinson $E J$, Kingston $R, O$ wen JJT, van Ewijk $W$ (1985) Stromal cell types in the developing thymus of the normal and nude mouse embryo. Eur J Immunol 15:675-681

12. Boyd RL, Tucek CL, Godfrey ID, Izon DJ, Wilson TJ, Davidson NJ, Bean AGD, Ladyman HM, Ritter MA, Hugo P (1993) The thymic microenvironment. Immunol Today 14:445-459

13. Shores EW, van Ewijk W, Singer A (1991) Disorganization and restoration of thymic medullary epithelial cells in $\mathrm{T}$ cell receptor-negative SCID mice: evidence that receptor-bearing lymphocytes influence maturation of the thymic microenvironment. Eur J Immunol 21:1657-1661

14. van Ewijk W, Shores EW, Singer A (1994) Crosstalk in the mouse thymus. Immunol Today 15:214-217

15. Surh CD, Ernst B, Sprent J (1992) Growth of epithelial cells in the thymic medulla is under control of mature T cells. J Exp Med 176:611-616

16. Shores EW, van Ewijk W, Singer A (1994) Maturation of medullary thymic epithelium requires thymocytes expressing 
fully assembled $\mathrm{CD}^{-} \mathrm{TCR}$ complexes. Int Immunol 6:1393-1402

17. Penit C, Lucas B, Vasseur F, Rieker T, Boyd RL (1996) Thymic medulla epithelial cells acquire specific markers by post mitotic maturation. Dev Immunol 5:25-36

18. Röpke C, van Soest P, Platenburg PPP, van Ewijk W (1995) A common stem cell for murine cortical and medullary thymic epithelial cells? Dev Immunol 4:149-156

19. Wang B, Levelt C, Salio M, Zheng D, Sancho J, Liu CP, She J, Huang M, Higgins K, Sunshine MJ, Eichmann K, Lacy E, Lonberg N, Terhorst C (1995) Over-expression of CD3 $\varepsilon$ transgenes blocks $\mathrm{T}$ Iymphocyte development. Int Immunol 7:435-448

20. Kawamoto H, Ohmura K, Hattori N, Katsura $Y$ (1997) $\mathrm{H}$ emopoietic progenitors in the murine fetal liver capable of rapidly generating $T$ cells. J I mmunol 158:3118-3124

21. Fehling $\mathrm{HJ}$, von Boehmer $\mathrm{H}$ (1997) Early $\alpha \beta$ T cell development in the thymus of normal and genetically altered mice. Curr Opin Immunol 9:263-275

22. van Ewijk W, Holländer GA, Terhorst C, Wang B (1998) Crosstalk in thymic microenvironments. Stepwise development of thymic microenvironments in vivo is regulated by thymocyte subsets (submitted)

23. Wekerle H, Ketelsen U-P, Ernst M (1980) Thymic nurse cells: lympho-epithelial cell complexes in murine thymuses. Morphological and serological characterization. J Exp Med 151:925-944

24. Brelinska R, Warchol JB (1997) Thymic nurse cells: their functional ultrastructure. Microsc Res Tech 38:250-266

25. van Vliet $E$, Jenkinson EJ, Kingston $R, O$ wen JJT, van Ewijk W (1985) Stromal cell types in the developing thymus of the normal and nude mouse embryo. Eur J Immunol 15:675-681

26. Germeraad WTV, Kawamoto $\mathrm{H}$, Jiang YF, Katsura $Y$, van Ewijk W (1998) Crosstalk in thymic microenvironments. Development of thymic microenvironments in vitro is regulated by thymocyte subsets and oxygen (submitted)

27. Jenkinson EJ, Franchi LL, Kingston R, O wen JJ (1982) Effect of deoxyguanosine on lymphopoiesis in the developing thymus rudiment in vitro: application in the production of chimeric thymus rudiments. Eur J Immunol 12:583-587

28. Godfrey DI, Kennedy J, Suda T, Zlotnik A (1993) A developmental pathway involving four phenotypically and functionally distinct subsets of $\mathrm{CD}^{-} \mathrm{CD}^{-} \mathrm{CD}^{-}$triple negative adult mouse thymocytes defined by CD44 and CD25 expression. J Immunol 150:4244-4245

29. Shortman K, Egerton M, Spangrude GJ, Scollay R (1990) The generation and fate of thymocytes. Semin Immunol 2:2-12 